\title{
Seroreversion of the serological tests for syphilis in the newborns born to treated syphilitic mothers
}

\author{
Sung Nam Chang, Kee-Yang Chung, Min-Geol Lee, Jung Bock Lee
}

\begin{abstract}
Backround-IgG antibodies from mothers adequately treated for syphilis can cross the placenta and appear in the sera of healthy newborns without infection. In such infants, a false diagnosis of congenital syphilis is often made. We have designed a retrospective survey to determine the time of seroreversion of the serological tests for syphilis (STS) in uninfected newborns born to mothers who were adequately treated for syphilis. Materials and Methods-Fifty two seropositive, untreated newborns born to 51 mothers treated for syphilis were studied. The newborns were followed at 1,3 , 6, 9, and 12 months of age until seroreversion was detected. The VDRL test was followed until 12 months in 12 of the 22 newborns who were positive at birth, the TPHA in 21 of the 46 newborns, and the FTA-ABS test in 22 of the 48 newborns.

Results-In the first serological tests done within 1 month after birth, the VDRL was positive in 22 newborns (42\%), the TPHA in $46(88 \%)$, and FTA-ABS in $48(92 \%)$. The VDRL seroreverted within 6 months after birth in $84 \%$, and within 1 year in $100 \%$. The TPHA test seroreverted in $95 \%$ within 1 year after birth. The FTA-ABS test seroreverted in $100 \%$ within 1 year after birth.

Conclusions-In most seropositive, untreated newborns born to treated mothers the VDRL became negative within 6 months after birth and the TPHA and FTA-ABS within 1 year. This result is consistent with current Centers for Disease Control (CDC) guidelines. However, although the CDC guidelines are adequate in general, we think that some revision is desirable concerning the IgM test and combination of the test results in order to rule out congenital syphilis in seropositive, nonsymptomatic newborns born to the treated mothers.
\end{abstract}

(Genitourin Med 1995;71:68-70)

Dermatology, Yonsei University College of Medicine, Seoul,

Korea

S N Chang

K-Y Chung

M-G Lee

J B Lee

Correspondence to: Jung Bock Lee, M.D., Department of

Dermatology, Yonsei

University College of

Medicine, C.P.O. Box 8044, Seoul, Korea.
During pregnancy Treponema pallidum can cross the placenta and cause intrauterine infection of the fetus. On the basis of the data reported previously, almost half of the infants born to mothers with active syphilis develop the disease, one quarter die in utero, and another quarter are not infected. ${ }^{1}$ Treatment of the syphilitic mothers with penicillin is very effective, preventing prenatal syphilis in 95 to $98 \%$ of the infants. ${ }^{2-5}$

IgG antibodies of adequately treated mothers can cross the placenta and appear in the sera of healthy newborns without infection. In such infants, a false diagnosis of congenital syphilis is often made and, owing to the limited availability of the $19 \mathrm{~S}(\operatorname{IgM})-\mathrm{FTA}$ test,${ }^{6}$ its confirmation is difficult. According to the guidelines by the Centers for Disease Control (CDC), ${ }^{7}$ if nontreponemal antibodies do not disappear by the sixth month of age, the infant should be treated for congenital syphilis. Additionally, if treponemal antibodies do persist beyond the first year of life, the child should also be treated.

Although these guidelines are broadly accepted at present, the supporting data seem to be few. We have designed this retrospective survey to determine the time of seroreversion of the STS in the uninfected newborns born to the treated mothers.

\section{Materials and methods}

In Yonsei Medical Centre, Seoul, Korea, the venereal disease research laboratory (VDRL) test is performed on maternal sera at the first prenatal visit, during the third trimester, and at delivery. The VDRL test is titred and fluorescent treponemal antibody-absorption (FTA-ABS) test is performed on all VDRLreactive sera. The serology is considered positive if both the VDRL and FTA-ABS tests are reactive. In addition, the Treponema pallidum haemagglutination assay (TPHA) and 19S(IgM)-FTA test are performed on all seropositive sera. All mothers who are diagnosed as having syphilis are treated with penicillin irrespective of the stage of pregnancy unless the patient is allergic to penicillin.

The VDRL, rapid plasma reagin (RPR), FTA-ABS, TPHA, and 19S(IgM)-FTA tests are performed on all the sera of neonates at 5 to 7 days of age whose mother was treated for syphilis.

In this study, mother-newborn pairs were identified by reviewing the charts of all the mothers treated for syphilis before delivery from January 1982 to November 1992. Forty nine mothers were considered treated as they had received penicillin at least 1 month before delivery. Two cases who had received tetracycline before pregnancy due to penicillinallergy were also considered treated.

The newborns' charts were reviewed to determine whether symptoms, signs, or labo- 
ratory tests indicated that congenital syphilis was present at birth. The newborns diagnosed as having congenital syphilis were excluded from this study. The diagnosis of congenital syphilis was based on the guidelines established by the CDC. ${ }^{7}$

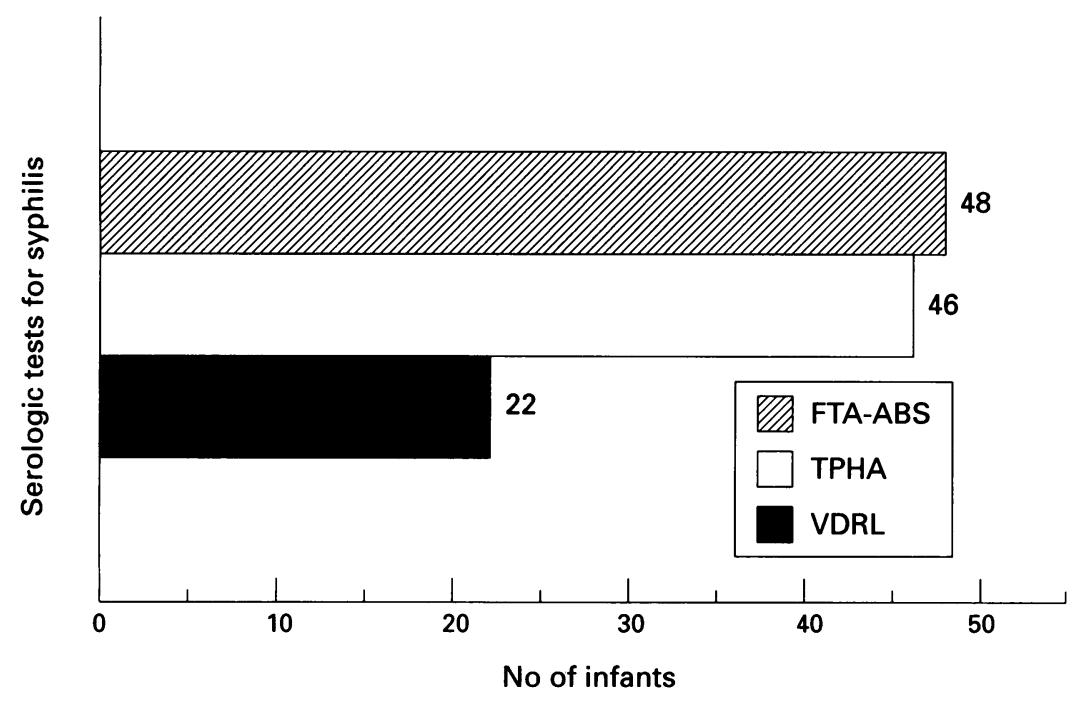

Figure 1 The number of infants with positive VDRL, TPHA, or FTA-ABS test within 1 month after birth among total 52 infants.

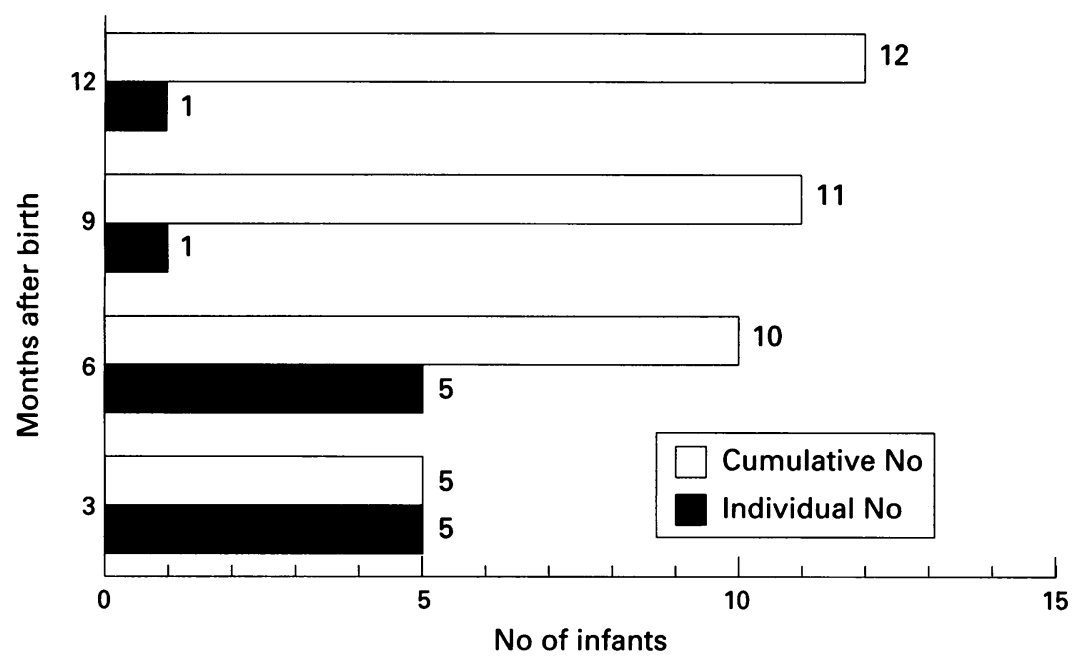

Figure 2 The number of seroreversions of the VDRL test during 12 months after birth among the 12 infants followed by the VDRL test.

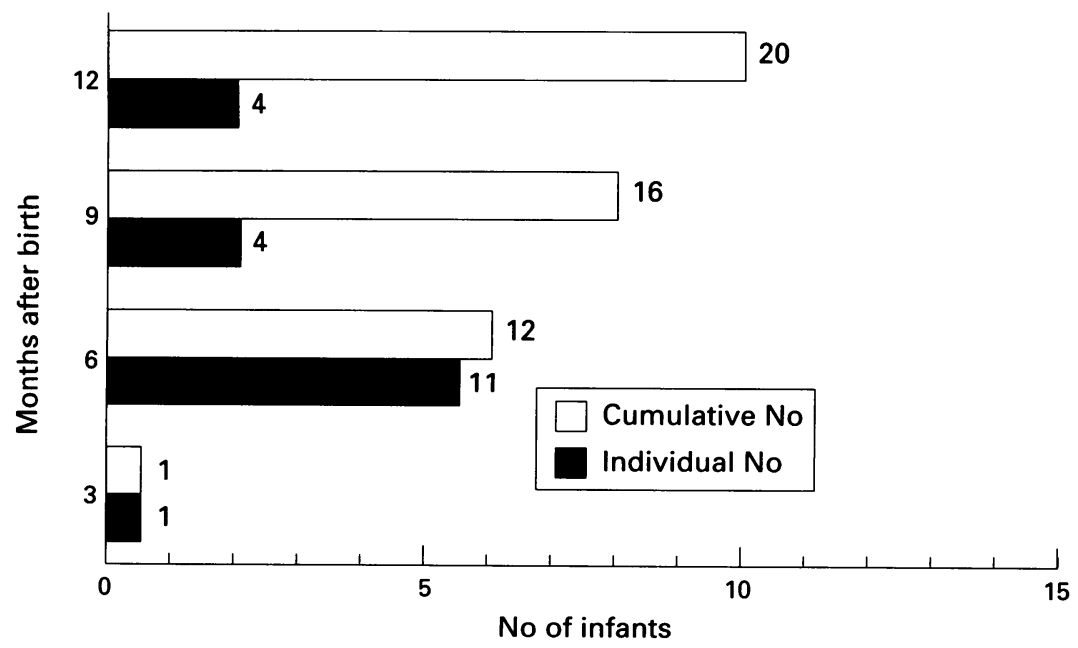

Figure 3 The number of seroreversions of the TPHA test during 12 months after birth among the 21 infants followed by the TPHA test.
Finally, 52 seropositive, untreated newborns born to 51 treated mothers were identified. The newborns were followed at $1,3,6$, 9 , and 12 months of age until seroreversion was detected. We were able to follow the VDRL until 12 months only in 12 of the 22 newborns who were positive at birth, the TPHA in 21 of the 46 newborns, and the FTA-ABS test in 22 of the 48 newborns.

\section{Results}

Treated mothers

Of the 51 mothers who were included in this study, 49 mothers were treated with penicillin and the remaining 2 with tetracycline before pregnancy due to penicillin allergy. The syphilitic mothers in this study were treated with benzathine penicillin $G$, doses ranging from $2 \cdot 4$ million to $7 \cdot 2$ million units, as recommended by the CDC. ${ }^{7}$ There were two mothers with primary syphilis, four with secondary syphilis, eight with early latent syphilis, eight with late latent syphilis, one with congenital syphilis, and 28 with indeterminate syphilis. Ten mothers received the treatment before pregnancy, 14 in the first trimester, 18 in the second trimester, and 9 in the third trimester.

\section{Newborns at birth}

Of the newborns born to the treated mothers, 52 were considered to be uninfected. Only one newborn who was excluded from our study was diagnosed as having congenital syphilis. This newborn had a 19S(IgM)-FTA test even though there were no clinical symptoms or signs.

In the first serological tests done within 1 month after birth the VDRL was positive in 22 newborns (42\%) the TPHA in $46(88 \%)$, and the FTA-ABS in 48 (92\%) (fig 1).

Infants followed up until 12 months of age Of the 12 infants followed with a VDRL, 5 $(42 \%)$ became seronegative at 3 months of age, $5(42 \%)$ at 6 months, $1(8 \%)$ at 9 months, and 1 (8\%) at 12 months, (fig 2). Therefore, the VDRL became seronegative within 6 months after birth in $84 \%$, and within 1 year in $100 \%$.

Of the 21 infants followed with a TPHA test, 1 infant $(5 \%)$ became negative at 3 months of age, $11(52 \%)$ at 6 months, 4 $(19 \%)$ at 9 months, $4(19 \%)$ at 12 months, and only $1(5 \%)$ remained positive until 12 months (fig 3). Therefore, the TPHA test became negative in $95 \%$ within 1 year.

Of the 22 infants followed with an FTAABS test, $1(5 \%)$ became negative at 3 months, $13(59 \%)$ at 6 months, $4(18 \%)$ at 9 months, and $4(18 \%)$ at 12 months of age (fig 4). Therefore, the FTA-ABS test became negative in all the infants within 1 year.

\section{Discussion}

The treatment of choice for syphilis in pregnancy is penicillin as it readily crosses the placenta and successfully treats the fetus as well as the mother. The treatment is very effective, preventing prenatal syphilis in 95 to $98 \%$ of 


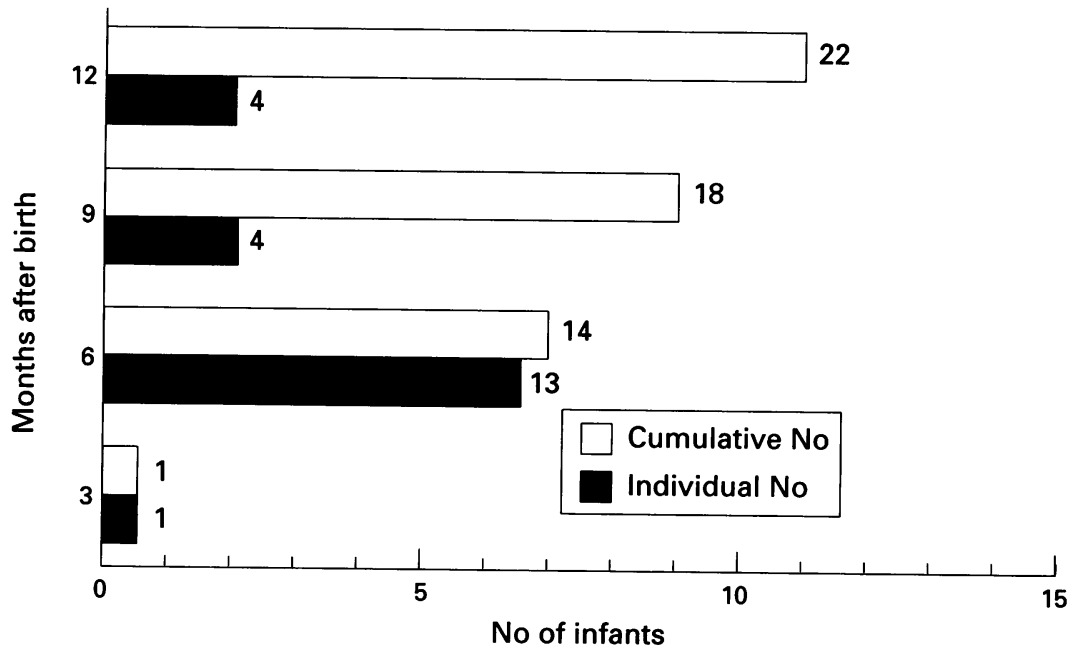

Figure 4 The number of seroreversions of the FTA-ABS test during 12 months after birth among the 22 infants followed by the FTA-ABS test.

the infants..$^{2-5}$ However, the risk of congenital syphilis in the neonates, even though their mothers have been treated with penicillin, cannot be completely ruled out. Therefore, the neonates born to the treated mothers should be evaluated for syphilis despite the low possibility of infection. The infected neonates born to the mothers treated during pregnancy were reported to have a milder form of congenital syphilis than the neonates born to the untreated mothers. ${ }^{8}$ Most infants with congenital syphilis are asymptomatic, and even when manifestations develop, they are often subtle and nonspecific. ${ }^{9-10}$

A definite diagnosis of congenital syphilis in a newborn requires the demonstration of $T$ pallidum in a lesion by dark field examination, immunofluorescence, or histological examination (such as with silver stain). However, as most of the newborns born to the treated mothers usually do not have skin lesions, the diagnosis of congenital syphilis is more dependent upon the STS rather than the demonstration of $T$ pallidum and classic symptoms. The main drawback of the STS is that the responding antibodies are easily found in healthy newborns because IgG antibodies can freely cross the placenta. In the STS done within 1 month after birth in our study, VDRL test was positive in $42 \%$ of the 52 newborns born to the treated mothers, TPHA in $88 \%$, and FTA-ABS test in $92 \%$. In a recently adopted diagnostic criterion ${ }^{7}$ for congenital syphilis, a newborn's VDRL titre should be four times as great as that of the maternal titre at delivery. But when a newborns's titre is the same as or even lower than the maternal titer, it is difficult to make a diagnosis of congenital syphilis solely on the basis of the STS results. Morever, owing to the limited availability of the $19 \mathrm{~S}(\operatorname{IgM})-\mathrm{FTA}$ test, which has very high sensitivity and specificity, ${ }^{6}$ the confirmation of congenital syphilis is difficult. In such cases, the CDC recommends to follow closely the seropositive, untreated infants at $1,2,3,6$, and 12 months after birth. ${ }^{7}$ In the absence of infection, nontreponemal antibody titres should decrease gradually and become nonreactive by 6 months of age. If the titres are found to be stable or increasing, the child should be reevaluated and fully treated. In addition, treponemal antibodies may be present up to 1 year in the absence of infection. If they are present beyond 1 year, the infant should be treated for congenital syphilis.

Although these guidelines are broadly accepted at present, the supporting data are not available concerning the follow-up of the newborns born to the treated syphilitic mothers. We have followed the seropositive, untreated infants until 12 months of age and found that $84 \%$ of the infants became seronegative within 6 months of age in the VDRL test, and $95 \%$ in TPHA and $100 \%$ in FTA-ABS within 1 year. Two infants had a positive VDRL beyond 6 months of age but as they had no clinical evidences of congenital syphilis and persistently negative $19 S(\operatorname{IgM})$ FTA, we did not treat them as recommended by the CDC. However, each of them turned out to show seroreversion at 9 and 10 months after birth. We also did not treat an infant who had a persistently positive TPHA beyond 12 months of age. He was regarded as not being infected as the VDRL and the FTAABS had already became negative.

In summary, most seropositive, untreated newborns born to the treated mothers became seronegative within 6 months after birth for the VDRL and within 1 year for the TPHA and FTA-ABS. Our results are consistent with the CDC guidelines. However, we documented three infants who had a persistently positive VDRL and TPHA test within the periods of diagnostic criteria suggested by the CDC (6 months of age for the VDRL and 12 months for the TPHA).

Although the CDC guidelines are adequate in general, we think that some revision is desirable concerning the IgM test and combination of the test results in order to rule out congenital syphilis in seropositive, nonsymptomatic newborns born to treated mothers. If the newborns born to treated mothers have positive STS for longer than 6 months, we recommend that decision for the treatment should be made more carefully with consideration of the findings of other STS, particularly 19S(IgM)-FTA test.

1 Danbolt $\mathrm{N}$, et al. The Oslo study of untreated syphilis: a restudy of the Boeck-Brunsgaard material concerning the fate of syphilitics who receive no specific treatment. Acta Derm Venereol (Stockh) 1954;34:34-8.

2 Ingraham NR, Beerman $H$. The present status of penicillin in the treatment of syphilis in pregnancy and infantile congenital syphilis. Am $\mathcal{F}$ Med Sci 1950;219:433-42.

3 Cole HN, Plotke F, Thomas FW, Jenkins KH. Penicillin in the treatment of syphilis in pregnancy. $\mathscr{f}$ Vener Dis Inform 1949;30:95-100.

4 Jackson FR, Vanderstoep EM, Knox JM, Desmond MM, Moore MB. Use of aqueous benzathine penicillin $\mathrm{G}$ in the treatment of syphilis in pregnant women. $A m$ Obstet Gynecol 1962;83:1389-92.

5 Hardy JB, Hardy PH, Oppenheimer EH, Ryans SJ, Sheff $\mathrm{RN}$. Failure of penicillin in a newborn with congenital syphilis. $¥ A M A$ 1970;212:1345-49.

6 Müller F. Der 19S(IgM)-FTA-ABS Test in der Serodiagnostik ${ }_{\text {der }}$ Syphilis, Test in der Fehlermöglichkeiten und diagnostische Aussage. Immun Infekt 1982;10:23-34.

7 Centers for Disease Control. 1993 sexually transmitted disease treatment guidelines. MMWR 1993;42(RR14):27-46.

Roca Gonzalez AM, Roldan Ros Am, Lopez Santiveri A, et al. Congenital syphilis and syphilitic mothers. Survey of the past 10 years. An Esp Pediatr 1992;37:135-9.

9 Kaufman RE, Jones OG, Blount JH, Weisner PJ Questionnaire survey of reported early congenital syphilis. Sex Transm Dis 1977;4:135-9.

10 Dorfman DH, Glaser JH. Congenital syphilis presenting in infants after the newborn period $N$ Engl 7 in 1990;323:1299-302. 